Implicit persuasion in medical decision-making: an overview of implicitly steering behaviors and a reflection on explanations for the use of implicitly steering behaviors

Ellen G. Engelhardt

Amsterdam Public Health Research Institute

Department of Epidemiology and Biostatistics

VU Medical Center

Medical faculty building F-wing, PO Box 7057, 1007 MB

Amsterdam, The Netherlands

e.engelhardt@nki.nl

Arwen H. Pieterse and Anne M. Stiggelbout

Department of Medical Decision Making

Leiden University Medical Center

J10-S, PO Box 9600, 2300 RC

Leiden, The Netherlands.

a.h.pieterse@1umc.nl and a.m.stiggelbout@lumc.nl

Word count: 5,002 main text and 81 words abstract 


\begin{abstract}
About the authors
Ellen G. Engelhardt is postdoctoral researcher currently at the Division of Psychosocial Research and Epidemiology and the Division of Molecular Pathology at the Netherlands Cancer Institute in Amsterdam. Arwen H. Pieterse is associate professor at the Department of Medical Decision Making at the Leiden University Medical Center in Leiden. Anne M. Stiggelbout is professor at the Department of Medical Decision Making at the Leiden University Medical Center in Leiden.
\end{abstract}

\title{
Key publications
}

- Stiggelbout A.M., A.H. Pieterse and J.C. De Haes. 2015. "Shared decision making: Concepts, evidence, and practice". Patient Education and Counseling 98:1172-1179.

- Engelhardt EG, A.H. Pieterse, A. van der Hout, H.J. de Haes, J.R. Kroep, P. Quarles van Ufford-Mannesse, J.E. Portielje, E.M. Smets and A.M. Stiggelbout. 2016. "Use of implicit persuasion in decision making about adjuvant cancer treatment: A potential barrier to shared decision making”. European Journal of Cancer 66:5566. 


\begin{abstract}
If the arguments to support a recommendation are partly implicit, the free exchange of ideas between discussants can be hampered. In this paper, we will focus on the potential pitfall for clinicians when informing patients about treatment options: implicit persuasion. We will describe a set of implicitly persuasive behaviors observed during decision-making consultations, and reflect on how these behaviors could undermine efforts to stimulate patient participation in decision-making. We will also reflect on possible explanations for why clinicians exhibit such behaviors.
\end{abstract}

Keywords: implicit persuasion; unbalanced information provision; preference-sensitive decisions; shared decision making 


\section{Information provision is a key component of the decision-making process}

Medical decision-making is a complex process that requires an exchange of information between healthcare providers and patients. Patients (often) lack the bio-medical knowledge to be able to form the informed treatment preference needed to make a treatment decision. Therefore, they rely on their doctor to provide them with the information and advice based on bio-medical knowledge and clinical experience. The clinician needs information about the patients' goals and priorities regarding the management of their illness, to be able to help patients weigh the pros and cons of available options and to provide this advice.

The objective of decision-making consultations is for patients and clinicians to arrive at a treatment plan that is in line with the patient's goals and priorities. To achieve this, clinicians' need to 1) ascertain patients' goals and priorities regarding the management of their illness, and 2) provide them with information about the pros and cons of relevant options. Particularly when faced with preference-sensitive treatment decisions, i.e., decisions for which there is no best option from a medical perspective, it is crucial to go through these steps adeptly. In the context of such decisions, the Shared Decision Making approach (SDM) is widely advocated. SDM consists of four key steps (Stiggelbout et al. 2015:1172-1179), namely:

1. Creating choice awareness: Clinicians need to explicitly communicate to patients that a treatment decision needs to be made, and that the patient's input is indispensable.

2. Information provision: For the patient to be able to participate in the decision-making process, clinicians need to inform the patient about the pros and cons of all viable treatment options - including the option to forego treatment if relevant.

3. Weighing pros and cons: Clinicians need to support patients in weighing the pros and cons to determine which treatment option the patient prefers, or best fits the patient.

4. Decision-making: After deliberation between doctor and patient about the pros and cons, the final treatment decision is made. Here, it is not relevant whether the patient, the doctor or both together make the final treatment decision, key is that the patient's goals and priorities have been considered.

Incorporating these key principles of SDM in the consultation, if executed competently, safeguards that the final treatment decision is medically sound and in line with patients' goals and priorities. In this paper, we will focus on the second step of SDM - information provision. A potential barrier to patient participation is unbalanced information provision. Especially, when faced with preference-sensitive decisions, it is important that the information does not contain implicit cues suggesting that there is a right option - i.e., implicit persuasion. 


\subsection{Implicit persuasion: definition and its prevalence in clinical practice}

Even if clinicians do not verbalize a treatment recommendation or consciously steer patients towards a specific treatment option, their choices about what information to disclose, and the way this information is phrased, can suggest to patients that their doctor favors a specific option. Implicitly steering behaviors could persuade patients to consent to a treatment plan that does not match their goals and priorities.

To the best of our knowledge, Karnieli-Miller and Eisikovits (2009:1-8) were the first to systematically operationalize the construct implicit persuasion in the context of medical decision-making consultations. In our own work (Engelhardt et al. 2016:55-66), we used the framework created by Karnieli-Miller and Eisikovits as a starting point for a coding scheme to assess the use of implicit persuasion in oncological practice, specifically in the context of adjuvant systemic therapy for early-stage breast cancer. We observed multiple implicitly persuasive behaviors in all the 105 consultations we analyzed (median of five behaviors per consultation (range: 2-10)). The direction of the implicit steering was predominantly toward undergoing treatment. This is noteworthy since $53 \%$ of the patients in our study population had stage 1 disease for which foregoing treatment generally is an option that will not significantly increase the probability of an unfavorable (long-term) outcome.

To date, besides the work by Karnieli-Miller and Eisikovits and our group, there have not been other studies that have systematically analyzed the use of implicit persuasion in clinical practice. In many studies of doctor-patient communication implicitly persuasive behaviors have been described, but they were not identified as such (see for example Ziebland et al. 2015:3302-3312). Also, decision aids aiming to support the shared decision-making process have been shown to contain implicitly persuasive language (Molewijk et al. 2008:415420). Evidence from systematic analyses is limited, but it seems likely that the use of implicit persuasion might be a widespread phenomenon in medical practice. More research is needed to get better insights into the extent to which implicit persuasion permeates medical decision-making.

\subsection{Is implicit persuasion inherently wrong?}

Given that there are indications that implicit persuasion might be pervasive in medicine, it is worth considering whether this is a cause for concern. Implicit persuasion is innately an undesirable phenomenon. At its core, implicit persuasion violates the imperative of patient autonomy, and is primarily rooted in a failure by clinicians to meet their duty to inform patients fully and adequately. Even if the clinician believes that steering (or nudging) patients towards the treatment she believes is in the patients' best interest, it likely impedes efforts to 
stimulate patient participation in decision-making. Implicit persuasion is particularly concerning when there is no best treatment option, as then, unbeknownst to them, patients are being steered towards a specific treatment about which it is uncertain whether it is best for them. When there is no best option from a medical perspective, patients' treatment goals and priorities should be the driving force in decision-making. Implicit persuasion might lead patients to think that their doctor knows the right option. Due to the inherent imbalance in power in the doctor-patient relationship, patients might be reluctant to verbalize apprehensions they might have towards the implicit treatment recommendation. Patients could thereby (inadvertently) be persuaded to consent to a treatment that is not the most suitable for them. The discordance between their goals and priorities and the treatment they receive, could result in the treatment constituting an unnecessarily large burden on their quality of life, and could even lead to sub-optimal treatment adherence or early treatment discontinuation, which could in turn affect the course of their disease.

\section{Implicitly persuasive behaviors}

We will describe the implicitly persuasive behaviors observed during decision-making consultations in the context of adjuvant systemic therapy for early-stage breast cancer. All the examples described here are from our previously published study; the study design and population are described elsewhere in detail (Engelhardt et al. 2016:55-66). We will first describe the setting.

\subsection{Clinical context}

Six out of ten breast cancer patients diagnosed between 2006 and 2012 in the Netherlands received adjuvant systemic therapy after the surgical removal of the main breast tumor and any metastases present in the axillary lymph nodes (Saadatmand et al. 2015:h4901). Adjuvant systemic therapy consists of: (1) chemotherapy for six months up to one year, and/or (2) endocrine therapy (i.e., tamoxifen and/or aromatase inhibitors) for at least five years. Adjuvant systemic treatment may improve patients' disease-free and breast cancer-specific survival probability. On an individual level treatment benefit varies, with absolute benefits ranging between 1-15\% (Early Breast Cancer Trialists Collaborative Group 1998:930-942; Davies et al. 2013:805-816). It is important to note that adjuvant systemic therapy is a preventative treatment, i.e., it is given to patients to potentially treat disease that may or may not be present (i.e., micro-metastasis which cannot be detected yet). Breast cancer is a very heterogeneous disease, and it is not yet possible to select patients that might benefit from treatment. Clinical guidelines recommend offering adjuvant systemic therapy to patients if the expected absolute treatment benefit is 
minimally $3-5 \%$. That minimal absolute benefit also means that it is currently deemed acceptable to treat 25 patients to save one patient from dying due to breast cancer. Therefore, for this preference-sensitive treatment decision, it is imperative that patients carefully consider whether the treatment benefits outweigh the harms considering their goals and priorities.

\subsection{Category 1: Unbalanced presentation of benefits and side-effects}

A balanced presentation of the benefits and side-effects of treatment is a key component of information provision during consultations. This helps patients to form an idea of what undergoing treatment entails - how it can affect their daily lives. It is unrealistic to assume that patients who are not adequately informed are able to form a wellinformed opinion on whether the benefits of treatment outweigh the side-effects - whether a treatment option is worthwhile to them. We present below five implicitly persuasive behaviors relating to unbalanced information provision.

2.2.1 Minimal number of side-effects presented. Both adjuvant chemotherapy and endocrine therapy for breast cancer have numerous side-effects. If we only look at the side-effects occurring often (i.e., in 1-10\% of patients) to very often (i.e., in more than $10 \%$ of patients), each of the adjuvant systemic treatment regimens has more than a dozen side-effects. Therefore, we deemed the communication about side-effects in our study (Engelhardt et al. 2016:55-66), to be insufficient if fewer than five side-effects were communicated to patients, respectively. Admittedly, the cut-off of five is arbitrary, but (inter-) national clinical guidelines do not provide guidance on which side-effects minimally need to be communicated. Fewer than five side-effects were communicated in eight out of ten consultations. This held for both chemotherapy and endocrine therapy.

One important factor underlying the minimal communication of side-effects is the fact that information provision about side-effects was routinely delegated to specialized nurses and/or nurse practitioners. Oncologists regularly indicated - after the decision was made - that they would only briefly go through some important sideeffects, but patients would receive extensive information during the consultation with the nurse prior to the start of treatment. For example: "You will receive all the information [about the side-effects and procedures] from the specialized nurse later on... she has more time and will go into more details about chemotherapy." Nowadays, in most Dutch outpatient clinics, specialized nurses act as case managers during the diagnosis, treatment, and follow-up of patients. Having such a constant factor is good for patients and it is a positive development that the nurse will go through the information with the patient. From the moment they receive the diagnosis, patients face 
several (life-changing) decisions in short succession during a period that is emotionally and physically trying. However, it is important for patients to be made aware of both the benefits and harms of the treatment options prior to deciding about treatment.

Finally, in addition to meeting minimal requirements regarding the number of side-effects communicated, attention also needs to be paid to the quality of the description of the side-effects. We observed very brief and little informative discussions about the side-effects during the consultation. Frequently information provision about side-effects only consisted of the mention of the side-effect (see Example 1).

\section{HERE BOX WITH EXAMPLE 1}

\subsubsection{Emphasizing the benefits or downplaying the side-effects of treatment. Repeatedly emphasizing the} magnitude of the treatment effect whilst minimizing the side-effects or vice versa, unbalances information provision. We observed this behavior in half of the 105 consultations analyzed (Engelhardt et al. 2016:5566). For example, when oncologists stated at various points during the consultation that the probability of developing a recurrence without treatment is "quite substantial”, whilst also repeatedly stressing that "You might experience side-effects, but most patients get through treatment without too many problems". Such repeated emphasis on the pros or the cons could suggest to patients that the direction of implicit steering indicates the right treatment option. Arguably, it is reasonable to assume that to many patients the clinician's implied preference carries much weight. This could stifle patients' own critical appraisal of the treatment options, as well as a discussion during the consultation of the concerns patients might have. Such implicitly steering behaviors are especially undesirable if no explicit advice is given, and patients only have their impression of the oncologist's preference. Such impressions could be wrong, and if not verbalized, incorrect assumptions might end up playing a (significant) role in decision-making.

2.2.3 Minimizing the treatment's impact. Utterances by oncologists can (unintentionally) minimize patients' awareness of the negative impact that the treatment could have. This behavior was observed in two out of five of the endocrine therapy consultations, and it was observed in 8 out of 90 chemotherapy consultations (Engelhardt et al. 2016:55-66). Examples of such utterances are: "Every medication has side-effects. Paracetamol has sideeffects, letrozole [a type of endocrine therapy] also has them.", or "Endocrine therapy is a relatively innocuous treatment, you just have to take a little tablet every day." Even though the oncologist does not explicitly say so, 
these statements suggest that the treatment is not so important in the grand scheme of things, and denote a preference for treatment.

Interestingly, oncologists were inconsistent in their framing of a specific treatment, and the variation did not seem due to patient characteristics that would determine the suitability of a treatment for that patient. Generally, the impact of endocrine therapy was minimized (Engelhardt et al. 2016:55-66), but in consultations in which oncologists seemed keen for the patient to consider chemotherapy, they would bring up that endocrine therapy is not as harmless as people commonly think, especially in comparison with chemotherapy (see Example 2). Such seemingly different framing of treatment impact was observed in consultations by the same oncologist; thus, it was not explained by differences between oncologists.

\section{HERE BOX WITH EXAMPLE 2}

2.2.4 Emphasizing the ability to control the side-effects of the treatment. The side-effects of adjuvant systemic therapy, particularly chemotherapy, are notorious and feared by patients. Oncologists tended to emphasize that it was possible to prevent or control the side-effects of treatment. Frequently stressed was the ability to prevent or control chemotherapy-induced nausea (see Example 3 and 4). This behavior was observed in half of the consultations analyzed (Engelhardt et al. 2016:55-66). If that medication proved insufficient, it was possible to up the dosage or try another type. Thereby, it was implied that the patient had control over the severity and management of the side-effect.

\section{HERE BOX WITH EXAMPLE 3 \\ \& HERE BOX WITH EXAMPLE 4}

\subsubsection{Presenting the side-effects after the final treatment decision has been made. A key requirement to patient}

involvement in treatment decision-making is that they are made aware of both the benefits and harms of treatment prior to the final treatment decision being made. Informing patients about the side-effects after making the decision violates this premise. It also means that the treatment decision was mainly based on the expected benefits. Even if the clinician does not explicitly give a treatment recommendation, the fact that the clinician seemingly does not deem the side-effects relevant to discuss before a treatment decision is made, could give patients the impression that their doctor feels that the expected benefits are worthwhile despite the side-effects. 
In two-thirds of the consultations, oncologists provided patients with information about the side-effects of treatment after the treatment decision had been made (Engelhardt et al. 2016:55-66). Interestingly, in several consultations the way in which the oncologist phrased the purpose of the consultation gave the impression that the treatment decision had already been made prior to the consultation (see Example 5). This behavior will impede active participation by patients in treatment decision-making. Arguably, patients will only go against the perceived treatment decision if they have a strong preference that does not match that decision.

\section{HERE BOX WITH EXAMPLE 5}

\subsection{Category 2: Presenting treatment recommendations as authorized decisions}

A crucial step to any decision-making consultation is to make patients aware that there is a decision to be made and that they have a say in it. However, even if choice awareness is created, patients' perception of whether they have choices can be limited if the phrasing of treatment recommendations is too strong. Below we describe two behaviors that might limit patients' perception of the say they have in treatment decision-making because they lend the treatment recommendation a lot of weight.

2.3.1 Presenting treatment as an authorized decision. Presenting treatment as an authorized decision based on

'the guideline': In one out of three consultations, treatment recommendations were phrased as being based on the national or international guidelines (Engelhardt et al. 2016:55-66) (see Example 6).

Presenting treatment as an authorized 'we' decision: In eight out of ten consultations, treatment recommendations were phrased as being the result of consensus amongst experts, for example: "We are in favor of...", or “As a profession we have decided that..." (Engelhardt et al. 2016:55-66) (see Example 6).

Why should the two behaviors described above be a cause for concern? At first glance these are mere factual statements, but they might (unintentionally) limit patient participation in the decision-making process. Such phrasing suggests that a group of experts think a certain option is worthwhile. Even if the oncologist indicates that the recommendation is not binding and the patient is free to choose a different option that better suits her, patients might not feel comfortable declining the perceived best option. They may fear that they will not be able to forgive themselves if they go against the recommended course of action and subsequently experience a negative outcome. Oncologists might not be aware of the added weight their phrasing imparts to the recommendation. From the oncologists' perspective, such phrasing denotes current practice. Devising treatment recommendations is not a solo activity; recommendations are indeed based on discussions in multidisciplinary 
cancer team meetings, and are guided by those discussions and the guidelines. However, it is important to make it clear to patients that all the options presented to them are medically viable options - there is no right choice, and preferring a different option to the one recommended does not constitute taking an unnecessary risk.

It is important to note here that we do not argue that it is wrong for clinicians to provide patients with an explicit treatment recommendation. On the contrary, some patients might find it very useful. Ideally, patients' priorities and goals inform such recommendations, not solely the clinicians' clinical experiences and guidelines. However, the evidence suggests that patient participation during consultations is limited (Couët et al. 2015:54261; Kunneman et al. 2015:338-342). Thus, it is likely that recommendations commonly are the result of the clinician's own weighing of the pros and cons, and are not informed by the patient's views on the pros and cons of the treatment options. And, whilst on a group level the assumptions underlying the treatment recommendation might hold, they might not hold for an individual patient.

\section{HERE BOX WITH EXAMPLE 6}

\subsection{Category 3: Creating the illusion of decision control}

The final step in the consultation is that of making the treatment decision. In our consultations, the primary treatment decisions that needed to be made were whether to undergo chemotherapy and/or endocrine therapy. However, we have observed behaviors in consultations that (unintentionally) shifted the focus away from these primary treatment decisions to secondary decisions. For example, the patient could decide whether to start the most intensive chemotherapy scheme or a lighter version, not whether to start treatment. The focus of information provision was also on that secondary decision, whilst the main decision was implicitly made by their oncologist. The primary decision is likely to be based on the treatment recommendation agreed upon during the multidisciplinary cancer team meeting. However, that recommendation is agreed upon prior to the oncologist meeting the patient, and consequently it might not be in line with patients' goals and priorities. And once a decision has been made, it may be exceedingly difficult for patients to take the initiative to broach the subject of revisiting it.

2.4.1 From mild to serious treatment - a gradual decision. The various possible strategies for a specific treatment option (e.g., type of chemotherapy regime or total number of courses the regime will consist of) can be presented in such a way that the proposed course of treatment seems to be the least aggressive, or invasive, and consequently most appealing option. This behavior was observed in one in five consultations (Engelhardt et al. 
2016:55-66). For example: "In your case we propose a four-course chemotherapy regimen instead of the standard six-course regime, which only yields slightly less disease-free survival gain, but is easier to pull through". Due to this manner of presentation the focus is shifted from the primary decision (i.e., whether to undergo chemotherapy) towards a secondary decision (i.e., what intensity of chemotherapy to undergo). Again, the fact that the option not to undergo treatment is (perhaps unintentionally) not discussed, could suggest to patients that their oncologist does not think this option is worth considering.

2.4.2 Having one treatment implicitly tag along with another. A combination of concurrent or consecutive treatments is presented as a "package deal", but the focus in the decision-making process is on one of the treatments, and the second treatment is implicitly agreed to as part of the package. In the context of adjuvant systemic therapy for early-stage breast cancer, chemotherapy and endocrine therapy can be presented as such, but the focus of the consultation is on chemotherapy. It is implicitly assumed that by agreeing to start chemotherapy, the patient is agreeing to undergo the whole package, even though endocrine therapy has not been (extensively) discussed. In our analyses, this behavior was observed in about one in three consultations (Engelhardt et al. 2016:55-66). Technically, the decision about endocrine therapy can be revised once the chemotherapy is completed, but patients might not realize this, or might find it difficult to broach the subject if the oncologist does not bring it up.

2.4.3 The illusory power to decide. There are instances in which the main treatment decision (i.e., whether to start treatment) is implicitly made by the clinician, and patients are given control over a secondary decision. For example, the clinician could emphasize that if the side-effects become too much to bear, the patient decides whether to stop with treatment or switch to another type of the same treatment (see Example 7). The patient does not make the primary treatment decision, but by leaving the decision about terminating or switching treatment in the patient's hands, the illusion is created that she has decisional control. This behavior was observed in half of the consultations (Engelhardt et al. 2016:55-66).

\section{HERE BOX WITH EXAMPLE 7}

\subsection{Category 4: Persuading patients using (clinical) experience}

Clinicians can (inadvertently) steer patients towards a specific treatment option by (overly) emphasizing their prior experiences with comparable patients. Given all the uncertainty patients face from the moment they receive 
their diagnosis, examples from their doctor's clinical experience can be something to hold on to. Such experience can be very powerful for patients and can play an important role in their weighing of the treatment options. The phrasing of and context in which such experiences are brought up, can suggest to patients that in their case there is a best option, which in turn may limit patients' own weighing of the pros and cons of treatment with their own personal situation in mind.

2.5.1 Making assertions about the patients' personality. The oncologist verbalizes assertions about what the patient might or might not be able to handle, her ability to persevere, and her priorities in life in the context of treatment. We observed this behavior in one in ten consultations (Engelhardt et al. 2016:55-66). Examples of such assertions are: "Some people's mood changes somewhat [due to endocrine therapy]. That is very rare, you do not seem like the type to experience that, but it could happen" or "If you are the type of person that sees things as the glass is half full rather than the glass is half empty you will get through it all better. I think you are more the type of person that sees things as the glass is half full'. Such statements might suggest to patients that their doctor is trying to tell them that they should at least try the treatment, i.e., that the clinician thinks they need it.

2.5.2 Deterring vs. encouraging: using others as examples. Using clinical experiences of other patients (i.e., frightening or hopeful stories), clinicians can steer patients towards the course of treatment they favor. Phrases associated with this behavior are for example: "in my experience, most women..." or "I have never come across...". Such phrasing could impede patients' own evaluation of the pros and cons of the various treatment options. As a layperson, a patient will be unlikely to go against the oncologist's experience. We observed this behavior in one in ten consultations (Engelhardt et al. 2016:55-66).

2.5.3 Giving the impression that undergoing or foregoing treatment is quite unusual. By emphasizing that it is very unusual for a patient with specific characteristics to undergo or forego a specific treatment option, clinicians suggest that considering another option is not a good idea from a medical perspective. For example: "It is unheard of for patients with HER2-positive disease like yours to forego chemotherapy and trastuzumab". It would take a patient with strong convictions, who is not afraid to carry the responsibility for the potentially 
negative consequences of her choice, to go against the option her clinician seems to prefer. Most patients might not feel comfortable going against what the clinical expert(s) recommend. We observed this behavior in one in ten consultations (Engelhardt et al. 2016:55-66).

\section{Reflection on potential explanations for the use of implicit persuasion}

In the previous sections, we introduced the construct of implicit persuasion, presented implicitly persuasive behaviors, and discussed for each behavior why and how it might derail attempts to involve patients in treatment decision-making. Here, we will reflect on potential explanations for the use of implicit persuasion. Based on our analysis of consultations (Engelhardt et al. 2016:55-66), we identified five factors we believe play a role in the use of implicit persuasion in the context of decision-making consultations.

\subsection{Attempts to prevent information overload}

An inexorable difficulty to information provision in clinical practice is that it is usually impossible to discuss all potential benefits and harms of the treatment options with patients. Clinicians must make choices about what information they disclose to patients, as providing too much information might overwhelm patients, and render them unable to utilize the information efficiently (Tversky and Kahneman 1973:207-232).

In the absence of guidance, in for example clinical guidelines, the decision about what to disclose is likely to be based on what the clinician thinks is relevant for their patient to know. This can vary between clinicians, both fueled by the clinician's own weighing of the benefits and harms in general as well as their clinical judgement as to the added value for a specific patient. Indeed, significant variation in the information disclosed to patients has been reported in the oncology setting (see Pieterse et al. 2011:e251-e259; HolmesRovner et al. 2015: 999-1009; Kunneman et al. 2015: 39-43). This suggests that between clinicians the weight given to the various benefits and harms differs significantly. The disparity in information provided to patients is a serious issue. It can be argued that clinicians do not meet their duty to inform patients if patients are not minimally made aware of the core set of benefits and harms that is relevant to all. In other words, the minimum legal requirement for informed consent is not met.

Further, prior to the consultation, for example during the multidisciplinary cancer team meeting, a decision is made about which treatment options will be presented to patients. Decision-making in this phase is driven by clinical guidelines and by what clinicians deem most suitable given disease and patient characteristics, such as disease stage, tumor grade and patient age, and oncologists' experience with patients with similar 
characteristics. Patients' goals and priorities regarding the management of their disease does not play a role (Scherr et al. 2017:56-69; Hahlweg et al. 2015:e0139921). If, clinicians do not invest time in exploring what the patient's goals and priorities are during the decision-making consultation with patients, they will not be able to gauge whether their assumptions about what is in the patient's best interest aligns with the patient's actual personal situation. Failing to check these assumptions alongside unbalanced information provision can result in a mismatch between patient goals and preferences and the treatment option that is decided on. Indeed, there are many reports in the literature showing that adequate exploration of the patient's expectations or ideas about how their illness is to be managed is minimal during consultations with patients (Couët et al. 2015:542-61; Pieterse et al. 2011:e251-e259; Holmes-Rovner et al. 2015:999-1009; Henry et al. 2015:1757-68; Henselmans et al. 2017:625-633).

\subsection{Lack of awareness of the gap in knowledge between doctors and patients}

Clinicians are aware that they have greater bio-medical knowledge than patients (e.g., about the course of the disease with and without treatment), and it is reasonable to assume that the exchange of information during consultations focuses on bridging this knowledge gap. However, clinicians might not realize that other aspects that are self-evident to them might not be obvious to (all) their patients. For example, clinicians frequently present treatment options and do not explicitly inform patients that no treatment is also a viable option (HolmesRovner et al. 2015:999-1009)., When not explicitly listed as an option, some patients might not realize that foregoing treatment is a viable option that does not have a significant detrimental effect on their long-term survival probabilities.

\subsection{Lack of awareness of own preferences}

It is unrealistic to assume that it is possible for clinicians to provide information that is in no way colored by their own preferences and beliefs about what is best for patients. Clinicians might not be aware that their choices regarding which information to disclose to patients can reflect their own preferences. And clinicians consistently (and logically) have been found to prefer the treatment in which they are specialized. For example, in the context of prostate cancer, Fowler and colleagues (2000:3217-3222) described that although urologists and radiation oncologists were aware that available treatment options generally did not outperform each other, they still tended to recommend the treatment they delivered themselves. Also, clinicians' beliefs about the benefit that makes treatment worthwhile are not always in line with the minimum treatment benefits used in guidelines to select 
patients with whom adjuvant treatment should be discussed (Engelhardt et al. 2015:956-959). Such preferences could influence how clinicians phrase information about treatment, for example, through downplaying the potential burden caused by the side-effects of treatment.

\subsection{Overcorrecting patients' perception of treatment burden}

Utterances by clinicians we would classify as being implicitly steering often seemed to be attempts to comfort patients, alleviate perceived anxiety, or correct misconceptions. For example, language minimizing treatment impact was often preceded by patients stating that they felt overwhelmed by it all, or expressing concern about whether they would be able to cope with treatment or anxiety about a specific side-effect (e.g., nausea, weight gain or mood changes). In the setting we studied, it seems that oncologists' method of addressing such patient utterances was to downplay the impact of treatment to reassure them or assuage their fears. Even though the intention is laudable, the key danger is overcorrecting and making it seem that for example the specific sideeffect the patient fears is of no consequence. This could cause patients to underestimate treatment impact, and agree to treatment without giving due consideration to the potential costs. Particularly in the case of adjuvant endocrine therapy, underestimation of treatment impact could be one of the driving forces behind the suboptimal adherence to treatment and early treatment discontinuation reported in the literature; up to as much as $50 \%$ of patients that start do not complete the full 5-year course of treatment (Hershman et al. 2010:4120-4128; van Herk-Sukel et al. 2010:843-851; Wouters et al. 2014:460-467).

\subsection{Overestimation of patient's assertiveness}

Since the paradigm shift from a paternalistic culture to patient-centered care, there is the notion that patients are increasingly assertive and will verbalize their views and opinions. This notion might cause too great a reliance on patients' assertiveness during consultations, and lead to clinicians underestimating how much weight their words carry. They could fail to realize that even if patients are assertive, in a relationship of dependency such as the doctor-patient relationship, particularly in life-threatening disease, it might be difficult for patients to go against the doctor's nudges. Going against their doctor's perceived preferences could also be difficult for patients as they might feel that possible negative consequences of their choice may rest solely on their shoulders.

\section{Conclusions}


Implicitly persuasive behaviors threaten clinician's ability to include patients in the decision-making process, even if the doctor intend to do so. Going forward, a key step is creating awareness amongst clinicians of behaviors that could inadvertently impede patient participation in treatment decision-making, for example by incorporating this subject in the medical curriculum and post-graduate courses. In addition to creating awareness, providing clinicians with tools to adequately implement the principles of SDM, especially how to explore patients' goals and priorities, and address patients' emotional needs, will prevent the use of many implicitly persuasive behaviors we have described here. It is also worth considering whether instructing clinicians in argumentation theory, specifically how to correctly present their arguments, could help improve their communication with patients. On a macro-level, much can be gained from the development of core lists of what information about treatment options is vital to communicate to all patients.

\section{References}

Couët Nicholas, Sophie Desroches, Hubert Robitaille, Hugues Vaillancourt, Annie Leblanc, Stéphane Turcotte, Glyn Elwyn and France Légaré. 2015. “Assessments of the extent to which health-care providers involve patients in decision making: A systematic review of studies using the OPTION instrument". Health Expectation 18:542-561.

Davies Christina, Hongchao Pan, Jon Godwin, Richard Gray, Rodrigo Arriagada, Vinod Raina, Mirta Abraham, Victor H. Medeiros Alencar, Atef Badran, Xavier Bonfill, Joan Bradbury, Michael Clarke, Rory Collins, Susan R. Davis, Antonella Delmestri, John F. Forbes, Peiman Haddad, Ming-Feng Hou, Moshe Inbar, Hussein Khaled, Joanna Kielanowska, Wing-Hong Kwan, Beela S. Mathew, Indraneel Mittra, Bettina Muller, Antonio Nicolucci, Octavio Peralta, Fany Pernas, Lubos Petruzelka, Tadeusz Pienkowski, Ramachandran Radhika, Balakrishnan Rajan, Maryna T. Rubach, Sera Tort, Gerard Urrutia, Miriam Valentini, Yaochen Wang, Richard Peto and the Adjuvant Tamoxifen: Longer Against Shorter Collaborative Group. 2013. "Long-term effects of continuing adjuvant tamoxifen to 10 years versus stopping at 5 years after diagnosis of oestrogen receptor-positive breast cancer: ATLAS, a randomised trial". Lancet 381:805-816.

Early Breast Cancer Trialists Collaborative Group. 1998. "Polychemotherapy for early breast cancer: an overview of the randomised trials". Lancet. 352:930-942. http://www.sciencedirect.com/science/article/pii/S0140673698033017. 
Engelhardt Ellen G., Hanneke C. de Haes, Cornelis J. van de Velde, Ellen M. Smets, Arwen H. Pieterse and Anne M. Stiggelbout. 2015. “Oncologists' weighing of the benefits and side effects of adjuvant systemic therapy: Has it changed over time?” Acta Oncologica 54:956-959. http://www.tandfonline.com/doi/pdf/10.3109/0284186X.2014.993478.

Engelhardt Ellen G., Arwen H. Pieterse, Anja van der Hout, Hanneke J. de Haes, Judith R. Kroep, Patricia Quarles van Ufford-Mannesse, Johanneke E. Portielje, Ellen M. Smets and Anne M. Stiggelbout. 2016. "Use of implicit persuasion in decision making about adjuvant cancer treatment: A potential barrier to shared decision making”. European Journal of Cancer 66:55-66.

Fowler, Jr Floyd J., Mary M. Collins, Peter C. Albertsen, Anthony Zietman, Diana B. Elliott and Michael J. Barry. 2000. "Comparison of Recommendations by Urologists and Radiation Oncologists for Treatment of Clinically Localized Prostate Cancer”. JAMA. 283:3217.

Hahlweg Pola, Jana Hoffmann, Martin Harter, Dominick L. Frosch, Glyn Elwyn and Isabelle Scholl. 2015. "In Absentia: An Exploratory Study of How Patients Are Considered in Multidisciplinary Cancer Team Meetings”. PLoSOne.;10:e0139921. http://www.ncbi.nlm.nih.gov/pmc/articles/PMC4595280/pdf/pone.0139921.pdf.

Henry Stephen G., Danielle Czarnecki, Valerie C. Kahn, Wen-Ying Chou, Angela Fagerlin, Peter A. Ubel, David R. Rovner, Stewart C. Alexander, Sara J. Knight and Margaret Holmes-Rovner. 2015. "Patientphysician communication about early stage prostate cancer: analysis of overall visit structure”. Health Expectations 18:1757-68.

Henselmans Inge, Hanneke W. Van Laarhoven, Jane Van der Vloodt, Hanneke C. De Haes and Ellen M. Smets. 2017. Shared decision making about palliative chemotherapy: A qualitative observation of talk about patients' preferences. Palliative Medicine 31:625-633.

Hershman Dawn L., Lawrence H. Kushi, Theresa Shao, Donna Buono, Aaron Kershenbaum, Wei-Yann Tsai, Louis Fehrenbacher, Scarlett L. Gomez, Sunita Miles and Alfred I. Neugut. 2010. "Early discontinuation and nonadherence to adjuvant hormonal therapy in a cohort of 8,769 early-stage breast cancer patients". Journal of Clinical Oncology 28:4120-4128.

Holmes-Rovner Margaret, Jeffrey S. Montgomery, David R. Rovner, Laura D. Scherer, Jesse Whitfield, Valerie C. Kahn, Edgar C. Merkle, Peter A. Ubel and Angela Fagerlin. 2015. “Informed Decision Making: Assessment of the Quality of Physician Communication about Prostate Cancer Diagnosis and Treatment”. Medical Decision Making 35:999-1009. 
Karnieli-Miller, Orit and Zvi Eisikovits. 2009. "Physician as partner or salesman? Shared decision making in real-time encounters". Social Science \& Medicine 69:1-8.

Kunneman Marleen, Corrie A. Marijnen, Monique C. Baas-Thijssen, Yvette M. van der Linden, Tom Rozema, Karin Muller, Elisabeth D. Geijsen, Anne M. Stiggelbout, Arwen H. Pieterse. 2015. "Considering patient values and treatment preferences enhances patient involvement in rectal cancer treatment decision making”. Radiotherapy and Oncology 117:338-342.

Kunneman Marleen, Corrie A.M. Marijnen, Tom Rozema, Heleen M. Ceha, Diana A.R.H. Grootenboers, Karin J. Neelis, Anne M. Stiggelbout and Arwen H. Pieterse. 2015. "Decision consultations on preoperative radiotherapy for rectal cancer: large variation in benefits and harms that are addressed". British Journal of Cancer 112:39-43.

Kunneman Marleen, Arwen H. Pieterse, Anne M. Stiggelbout and Corrie A. Marijnen. 2015. "Which benefits and harms of preoperative radiotherapy should be addressed? A Delphi consensus study among rectal cancer patients and radiation oncologists". Radiotherapy and Oncology 114:212-217.

Molewijk Albert, Anne M. Stiggelbout, Wilma Otten, Heleen M. Dupuis and Job Kievit. 2008. "First the facts, then the values? Implicit normativity in evidence-based decision aids for shared decision-making". Zeitschrift für Evidenz, Fortbildung und Qualität im Gesundheitswesen 102:415-420.

Pieterse Arwen H., Inge Henselmans, Hanneke C. de Haes, Caro C. Koning, Elisabeth D. Geijsen and Ellen M. Smets. 2011. "Shared decision making: prostate cancer patients' appraisal of treatment alternatives and oncologists' eliciting and responding behavior, an explorative study”. Patient Education and Counseling 85:e251-e259.

Saadatmand Sepideh, Reini Bretveld, Sabine Siesling and Madeleine M. Tilanus-Linthorst. 2015. "Influence of tumour stage at breast cancer detection on survival in modern times: population based study in 173,797 patients”. British Medical Journal 351:h4901.

Scherr Karen A., Angela Fagerlin, Timothy Hofer, Laura D. Scherer, Margaret Holmes-Rovner, Lillie D. Williamson, Valerie C. Kahn, Jeffrey S. Montgomery, Kirsten L. Greene, Biqi Zhang and Peter A. Ubel. 2017. "Physician Recommendations Trump Patient Preferences in Prostate Cancer Treatment Decisions". Medical Decision Making 37:56-69.

Stiggelbout Anne M., Arwen H. Pieterse and Hanneke C. De Haes. 2015. "Shared decision making: Concepts, evidence, and practice”. Patient Education and Counseling 98:1172-1179. 
Tversky Amos and Daniel Kahneman. 1973. "Availability: A heuristic for judging frequency and probability”. Cognitive Psychology 5:207-232.

van Herk-Sukel Myrthe P., Lonneke V. van de Poll-Franse, Adri C. Voogd, Grard A. Nieuwenhuijzen, Jan Willem Coebergh and Ron M. Herings. 2010. "Half of breast cancer patients discontinue tamoxifen and any endocrine treatment before the end of the recommended treatment period of 5 years: a population-based analysis". Breast Cancer Research and Treatment 122:843-851.

Wouters Hans, Anne M. Stiggelbout, Marcel L. Bouvy, Gemma A. Maatman, Erica C. Van Geffen, Robbert Vree, Johan W. Nortier, Liset van Dijk. 2014. "Endocrine therapy for breast cancer: assessing an array of women's treatment experiences and perceptions, their perceived self-efficacy and nonadherence". Clinical Breast Cancer 14:460-467.

Ziebland Sue, Alison Chapple and Julie Evans. 2015. "Barriers to shared decisions in the most serious of cancers: a qualitative study of patients with pancreatic cancer treated in the UK". Health Expectations 18:3302-12. 


\section{FIGURES CONTAINING QUOTES}

\section{Example 1: Excerpt from a consultation with a 62-year-old patient with stage 2 breast cancer}

Oncologist: The chemotherapy itself is not such a problem... of course there are some side-effects that need to be discussed calmly. For that you will get an appointment with our nurse, who will explain the side-effects and she will also give you some information on paper. If we only briefly discuss the side-effects, I think most important to mention is that it causes fatigue and a little bit of nausea.

Patient: Oh, how awful, I find that [the nausea] so awful.

Oncologist: The nausea can usually be reduced with medication.

Patient: Okay.

Oncologist: Generally, it is fine...... and there is hair loss

Patient: Is there always hair loss?

Oncologist: Yes, with this chemotherapy there is.

Patient: Yes? ....... Okay

Oncologist: Yes...

Patient: Well, I know what I am up against with this one [the chemotherapy].

Oncologist: These are the major side effects, plus the fact that chemotherapy makes you more susceptible to infections. Your defenses will be down. That is why we also check a day or two before the chemotherapy is given. [Information about blood work done prior to each course of chemotherapy]

Patient: Uhm...

Oncologist: So that is important... it is important that you always get in touch if you get a fever. The nurse will also explain all this. But it is important that you get in touch because your immune system is compromised, and if you develop an infection your body will be less able to fight it. Fortunately, it hardly ever happens, but it could.

Patient: What a hassle...

Oncologist: So those are the important things. You always hear dramatic things about chemotherapy, and we will also tell you. Generally, things turn out well.

\section{Example 2: Quote from a consultation with a 67-year-old patient with stage 2 breast cancer}

Oncologist: Everyone always thinks that endocrine therapy, that is just little tablets, it's not such a big deal. But that is not the case. That is not the case. What I find particularly difficult is that if the side-effects of the endocrine therapy are greater than expected and if you decide to stop [the endocrine treatment], then you won't have had any treatment.

\section{Example 3: Quote from a consultation with a 56-year-old patient with stage 1 breast cancer}

Oncologist: You can expect nausea. Therefore, as a precaution we will give you medication against the nausea. I cannot promise you that you will not get nauseous, but that [whether you get nauseous] also depends a little bit on your attitude. Some people get nauseous if they think about the hospital. That we cannot do much about.

\section{Example 4: Quote from a consultation with a 70-year-old patient with stage 1 breast cancer}

Oncologist: Complaints such as nausea or vomiting are usually not so bad. Especially given the anti-nausea tablets we give nowadays, it is no longer the case that people get really... well are continuously vomiting. Really, vomiting is no longer... a part of it anymore. With the tablets, you should be able to prevent it [vomiting]. 
Example 5: Quote from a consultation with a 46-year-old patient with stage 2 breast cancer

Oncologist: After your surgery your case was discussed during the multidisciplinary cancer team meeting, as we call it... I am going to talk about the period after the radiotherapy, because you will now start with radiotherapy first. And about four weeks after the radiotherapy, you will start with the chemotherapy.

\section{Example 6: Quote from a consultation with a 51-year-old patient with stage 1 breast cancer}

Oncologist: We advise what the guideline says. The guideline says that it [chemotherapy] is for everyone, that is what has been agreed on, on a national level, for whom the expected treatment benefit is 5\% or more. Everyone [oncologists] thinks it is worthwhile. However, they are not the ones that have to undergo it, they are only the experts. It is up to you whether you think it is worthwhile or not.

\section{Example 7: Quote from a consultation with a 62-year-old patient with stage 1 breast cancer}

Oncologist: About the endocrine therapy tablets we often say, just try it. The side-effects really are not so bad that you cannot cope with them. And if you really find the hot flushes awful, if you find the misery too awful, then you can always stop, then the side-effects will go away. Uhm, ... it is not compulsory, but it is something about which we usually say ... just try it. 